\title{
Luminescence Properties of Thiol-Stabilized CdTe Nanocrystals
}

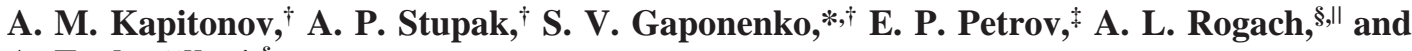 \\ A. Eychmiiller*,\$ \\ Institute of Molecular and Atomic Physics and B. I. Stepanov Institute of Physics, National Academy of \\ Sciences of Belarus, F. Skaryna Ave. 70, 220072 Minsk, Belarus, and Institute of Physical Chemistry, \\ University of Hamburg, Bundesstrasse 45, 20146 Hamburg, Germany
}

Received: June 28, 1999

\begin{abstract}
Thiol-capped CdTe nanocrystals with cubic zinc blende structure are synthesized in aqueous solution. Their steady-state and time-resolved luminescence characteristics are studied at room and liquid nitrogen temperatures. A strong exciton luminescence peak at $2.3 \mathrm{eV}$ dominates the emission spectrum of CdTe nanocrystals at room temperature, whereas the trap band centered at $2.0 \mathrm{eV}$ undergoes substantial temperature quenching. Luminescence excitation spectra reveal different channels leading to radiative recombination via either excitons or traps. The mean luminescence decay time of CdTe nanocrystals at room temperature decreases from 120 ns at $1.94 \mathrm{eV}$ to $20 \mathrm{~ns}$ at $2.43 \mathrm{eV}$. Luminescence decay kinetics of CdTe nanocrystals are strongly nonexponential and are described by extremely broad lifetime distributions lying within the range from a few hundred picoseconds to a few hundred nanoseconds.
\end{abstract}

\section{Introduction}

Semiconductor nanocrystals are currently being extensively studied in the context of their size-dependent photophysical and photochemical properties. The size of the nanocrystals in all three dimensions is less than the de Broglie wavelength of the electronic subsystem excitations. Therefore, many properties of these materials can be systematically described and understood in terms of quantum confinement effects. ${ }^{1-5}$ From the viewpoint of basic science, a nanocrystal is in an intermediate state of matter between molecule-like clusters and bulk crystals, and therefore offers a possibility to trace an evolution of electronic and optical properties of the matter from small atomic clusters to bulk solids. In the context of applications, nanocrystals can be efficiently used in novel light-emitting devices, optical transformers, photonic switches, nanoelectronic circuitry, and other applications. ${ }^{6-11}$

Though the basic properties of II-VI nanocrystals including electron-hole energy states and optical transition probabilities are well established and understood, ${ }^{12-14}$ there are a number of important issues relevant to primary photophysical and photochemical processes that are still under investigation. Photoluminescence properties of nanocrystals typically depend on a variety of parameters that do not affect the absorption properties. ${ }^{15}$ These parameters are surface structure, chemical environment, migration of carriers, and a number of guest-host effects. An understanding of nanocrystal properties beyond simplified quantum dot considerations is necessary for establishing photoluminescence mechanisms in real nanocrystals and the development of highly efficient light-emitting nanocrystalline devices.

In the present paper we report on steady-state and timeresolved photoluminescence properties of thiol-capped CdTe

\footnotetext{
Institute of Molecular and Atomic Physics; http://imaph.bas-net.by.

B.I. Stepanov Institute of Physics.

$\S$ University of Hamburg.

" Permanent address: Physico-Chemical Research Institute, Belarusian State University, 220080 Minsk, Belarus.
}

nanocrystals in aqueous solution. Unlike nanocrystals of CdS and CdSe whose structural and luminescence properties have been thoroughly examined and reviewed, 1,4,5,16,17 cadmium telluride nanocrystals are much less studied. Although several groups have performed the synthesis of CdTe nanocrystals in various environments, ${ }^{18-25}$ studies of their luminescence properties are rather fragmentary and preliminary.

\section{Experimental Section}

A. Sample Preparation and Characterization. The preparation and characterization of 1-thioglycerol-capped CdTe particles have been described in detail elsewhere (sample $\mathbf{c}$ in ref 24). Briefly, $22 \mathrm{~mL}$ of freshly prepared oxygen-free $0.05 \mathrm{M}$ NaHTe solution was added to $125 \mathrm{~mL}$ of a $0.013 \mathrm{M}$ nitrogen-saturated $\mathrm{Cd}\left(\mathrm{ClO}_{4}\right)_{2} \cdot 6 \mathrm{H}_{2} \mathrm{O}$ aqueous solution at $\mathrm{pH} 11.2$ in the presence of $0.5 \mathrm{~mL}$ of 1-thioglycerol as a stabilizing agent. The solution was heated to $96{ }^{\circ} \mathrm{C}$ and refluxed for $8 \mathrm{~h}$ to promote the growth of CdTe nanoparticles. The method of size-selective precipitation $^{26}$ was used to separate a CdTe nanoparticle fraction with relatively narrow $(<10 \%$ as confirmed by transmission electron microscopy) size distribution. CdTe nanoparticles precipitated by 2-propanol were redissolved in water and used for spectroscopic measurements. Powder X-ray diffractometry was performed using a powdered CdTe sample. A predominantly cubic zinc blende phase was derived from the diffractograms. The existence of a well-pronounced diffraction peak in the smallangle region further confirms the monodispersity of the sample. High-resolution transmission electron microscopy (HRTEM) indicates good crystallinity of the CdTe particles; Fourier analysis of high-resolution micrographs of single CdTe particles also revealed the cubic structure of the nanocrystallites. ${ }^{24}$ The mean particle size estimated from the HRTEM images and from the X-ray diffraction patterns of CdTe particles yields a value of about $2.4 \mathrm{~nm} .{ }^{27}$

B. Spectroscopic Measurements and Data Processing. Absorption spectra were recorded at room temperature on a Perkin-Elmer Lambda 14 UV-vis spectrophotometer. Lumi- 
nescence emission and excitation spectra and luminescence decay kinetics were measured at room and liquid nitrogen temperatures. Steady-state luminescence emission and excitation spectra were recorded on an SFL-1211A (SOLAR, Minsk, Belarus) spectrofluorometer with a cooled photomultiplier as a detector. The typical spectral resolution for both the excitation and emission monochromators was 3-4 $\mathrm{nm}$.

Time-resolved luminescence measurements were carried out on a PRA-3000 (Photochemical Research Associates, London, Ontario) spectrofluorometer in the time-correlated single photon counting mode with a wide-band cooled photomultiplier tube (PMT) used as a detector. An electric discharge in air (fwhm $\approx 2 \mathrm{~ns}$ ) at $20 \mathrm{kHz}$ repetition rate was used as an excitation source. Slit widths of monochromators of both excitation and emission channels were set to $16 \mathrm{~nm}$. Decay kinetics were recorded in $m=511$ time channels. Typically, $5 \times 10^{3}-2 \times$ $10^{5}$ counts in a peak channel (CPC) of the decay curve were collected.

C. Luminescence Decay Data Analysis. In the case when the finite duration of the impulse response function of a fluorometer cannot be neglected, an experimentally detected luminescence decay kinetics $d(t)$ is represented by a convolution of a "true" decay law $D(t)$ with the apparatus response function $R(t):^{28}$

$$
\int_{0}^{t} R\left(t-t^{\prime}\right) D\left(t^{\prime}\right) \mathrm{d} t^{\prime}=d(t)
$$

Two different methods of decay data analysis were used in this work. First, luminescence decays were analyzed using the model-based reconvolution using the conventional one- to threeexponential decay models and several nonexponential decay kinetics previously successfully applied to an analysis of luminescence decays of semiconductor nanocrystals (vide infra). The second-order local polynomial approximation of the apparatus response function ${ }^{29}$ was used in reconvolution routines. The quality of data fitting and the consistency of a decay model were judged on the basis of the visual inspection of plots of weighted residuals and their autocorrelation function, as well as on the basis of the values of the reduced chi squared $\chi^{2}$ and Durbin-Watson parameter (DW). ${ }^{28}$

Second, luminescence kinetics were analyzed using the approach based on the recovery of decay time distributions (see, e.g., ref 30) with minimum a priori assumptions on the luminescence decay law. In this case, the "true" luminescence decay is presented as originating from an (unknown) distribution of decay times $f(\tau)$

$$
D(t)=\int_{\tau_{\min }}^{\tau_{\max }} f(\tau) \exp (-t / \tau) \mathrm{d} \tau
$$

and the decay time distribution analysis reduces to the inversion of the quasi-Laplace transform (2). In the numerical treatment of decay data, the integral (2) is approximated by the exponential series $\sum_{i=1}^{n} f_{i} \exp \left(-t / \tau_{i}\right)$ with fixed decay times $\tau_{i}$ spanning the range $\left(\tau_{\min }, \tau_{\max }\right)$ and unknown preexponential factors $f_{i}$. Thus, discretization of eq 1 with regard for eq 2 leads to a system of linear equations

$$
\mathbf{K f}=\mathbf{d}
$$

where $\mathbf{K}$ is an $m \times n$-matrix combining the discretized convolution and Laplace transform kernels, $\mathbf{d}$ is an $m$-vector of raw decay data, and the $n$-vector $\mathbf{f}$ is the sought distribution of preexponential factors.

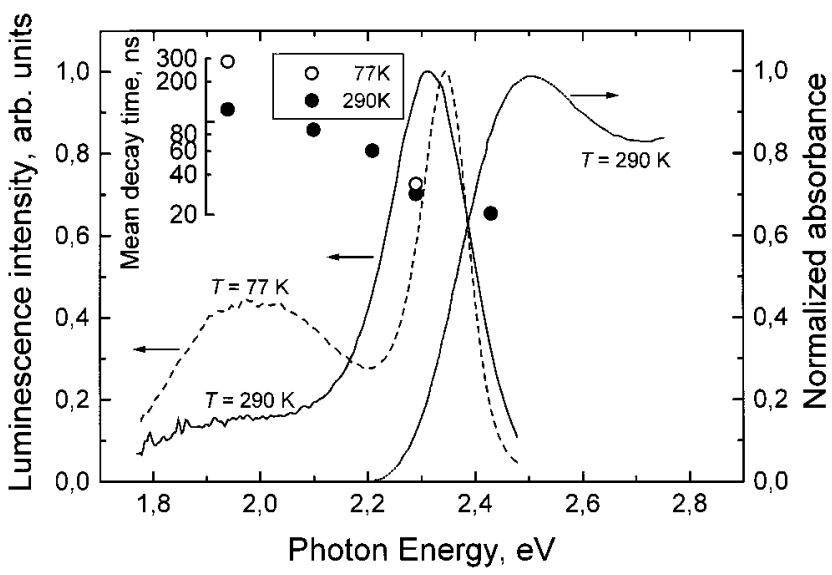

Figure 1. Normalized luminescence and absorption spectra of CdTe nanocrystals. Absorption was measured at room temperature and luminescence at both room (excitation at $480 \mathrm{~nm}=2.58 \mathrm{eV}$ ) and liquid nitrogen (excitation at $460 \mathrm{~nm}=2.69 \mathrm{eV}$ ) temperatures. The inset presents the spectral dependence of the mean luminescence lifetime at room (solid circles) and liquid nitrogen (open circles) temperatures.

The system of equations (3) approximates an integral equation of the first kind and is therefore severely ill-conditioned (see, e.g., ref 31). Therefore, a regularization method should be used for the recovery of a decay time distribution from luminescence decay kinetics. In the present work, luminescence decay data were analyzed using a Tikhonov-regularization-based routine, ${ }^{32}$ in which the regularization parameter is evaluated on the basis of a combination of a partial singular-value decomposition computed using the NIPALS algorithm ${ }^{33}$ and the generalized cross-validation approach. ${ }^{34}$ Decay time distributions were computed using $n=100$ exponential terms with decay times uniformly spaced on the logarithmic scale, which provides optimum resolution for inverting the Laplace transform. ${ }^{35}$

\section{Results and Discussion}

A. Steady-State Luminescence. Room-temperature absorption and luminescence spectra of CdTe nanocrystals and the luminescence spectrum recorded at liquid nitrogen temperature $(77 \mathrm{~K})$ are presented in Figure 1. The well-pronounced absorption at about $2.5 \mathrm{eV}$ is indicative of a rather narrow size distribution of CdTe nanocrystals. In the case of a $10 \%$ size distribution inherent in the samples investigated, the variation in the energy $E_{1 \mathrm{~S} 1 \mathrm{~S}}$ of the lowest excitonic transition in nanocrystals should lie in the range of $150-200 \mathrm{meV}$. However, the spectral width of the first absorption feature is about 500 meV. Therefore, this feature should consist of a series of unresolved electronic transitions of different kinds and cannot be associated with the lowest energy of the $\mathrm{E}_{1 \mathrm{~S} 1 \mathrm{~S}}$ transition alone. This agrees with the results of photophysical and photochemical spectral hole burning $25,36,37$ in an ensemble of II-VI crystallites, where a complex structure of the first absorption feature was revealed for nanocrystals in the strong confinement regime.

The luminescence spectra of CdTe nanocrystals consist of a sharp high-energy peak of excitonic luminescence with a wide low-energy band of emission from trap states. The contribution from trap luminescence decreases with increasing temperature. At $77 \mathrm{~K}$, the exciton luminescence band is centered at about $2.34 \mathrm{eV}$. This band is inhomogeneously broadened due to the size distribution of CdTe crystallites and fluctuations in their microenvironment. Its fwhm exceeds $100 \mathrm{meV}$ and the full width at the base of the band is in the range of $150-200 \mathrm{meV}$, which agrees well with the above-estimated variation in the lowest transition energy. The trap luminescence band spreads 


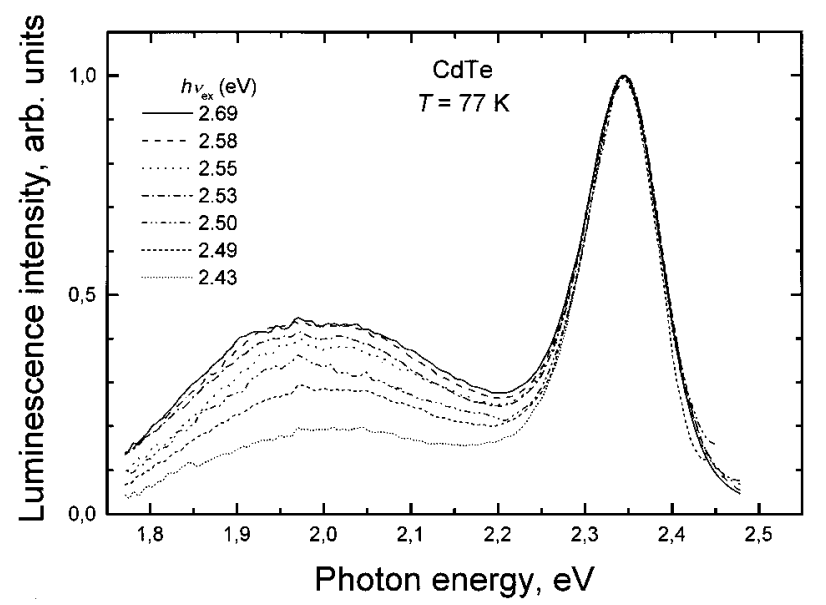

Figure 2. Luminescence of CdTe nanocrystals recorded for a set of excitation energies at $77 \mathrm{~K}$.

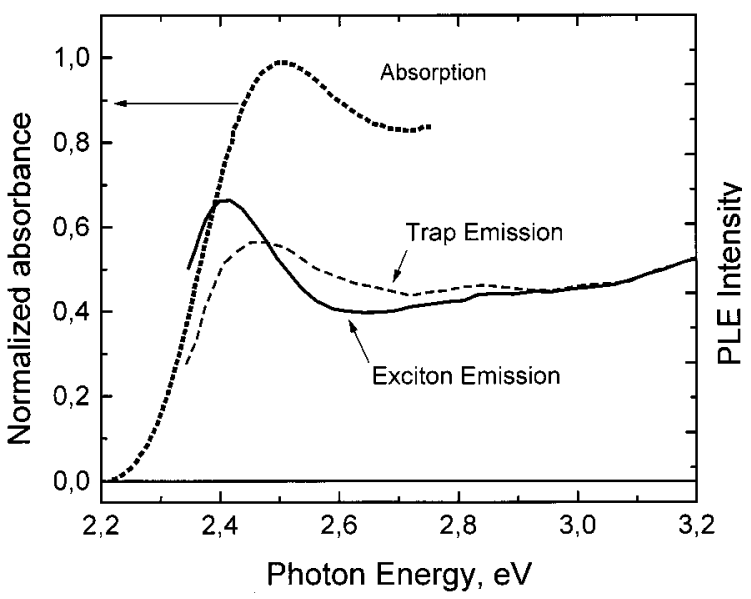

Figure 3. Room-temperature excitation spectra of trap and exciton emission in CdTe nanocrystals. The absorption spectrum is also displayed for reference.

from the exciton peak down to $\sim 1.7 \mathrm{eV}$, and its maximum at $77 \mathrm{~K}$ is located near $1.95-2.00 \mathrm{eV}$. Such a wide emission band indicates a wide energy distribution in the trap states, implying a variety of defects involved in light emission. The roomtemperature luminescence spectrum possesses the same features, namely, the sharp exciton luminescence peak and the wide traprelated band, which is less pronounced than at low temperature.

The evolution of the normalized luminescence spectra at 77 $\mathrm{K}$ of thiol-stabilized CdTe nanoparticles with the excitation energy is shown in Figure 2. The excitation wavelength was varied within the range of $460-510 \mathrm{~nm}(2.69-2.43 \mathrm{eV})$. No changes in the luminescence spectrum were detected in the vicinity of the exciton luminescence peak, even with the excitation within the high-energy emission tail. The low-energy part of the luminescence spectrum (which is characteristic of radiative recombination via defects) undergoes a dramatic change under the same conditions. When the excitation approaches the absorption edge, the relative contribution of emission from trap states decreases abruptly. A small deviation from this behavior occurs only in the vicinity of $2.55 \mathrm{eV}$.

Figure 3 shows the room-temperature excitation spectra for trap emission (dashed line) and excitonic emission (solid line) along with the absorption spectrum (dotted line). The excitation spectra have been normalized to provide the same shortwavelength intensity values. Detection energies were chosen at the maxima of the corresponding bands, i.e., $2.294 \mathrm{eV}$ for excitonic emission and $1.936 \mathrm{eV}$ for emission from trap states.
The latter value was chosen on the basis of the low-temperature data, where the trap band is more pronounced. Both excitation curves exhibit similar features, namely, a low-energy maximum. The fact that they do not coincide means that the absorbed photon should possess a higher energy to excite the trap emission as compared to the exciton luminescence. There are three possible explanations for this phenomenon.

First, smaller crystallites whose absorption edge lies at relatively higher energies due to stronger confinement could be responsible for virtually all emission from trap states, which was established for $\mathrm{Cd}_{3} \mathrm{P}_{2}$ nanoparticles. ${ }^{38}$ In fact, smaller nanocrystals have larger surface-to-volume ratio, and hence the probability of the appearance of trap states due to surface defects is also higher. Alternatively, if a nanoparticle with dimensions of several crystal lattice periods $(0.648 \mathrm{~nm}$ for zinc blendetype $\mathrm{CdTe}^{39}$ ) has a few defects (on the surface or in the volume), then the effective radius $a$ of this particle can decrease because of a decrease in the volume available for propagation of Bloch's waves. Such a scenario should be valid if defects distort the periodic lattice potential at least to the extent of one crystal unit cell. A rather small integral quantum yield of luminescence at room temperature $(\sim 3 \%)$ agrees with the assumption that a considerable part of the crystallites is defective.

Second, nanocrystals that differ in the quantum yield of intrinsic and trap emission may differ not only in size. For example, we could have two different kinds of particles in the solution: one exhibiting exclusively the exciton luminescence and the other having traps at the surface because of incomplete capping giving rise to the red-shifted luminescence. Direct observation of the single-particle luminescence should verify the possibility of coexistence of different kinds of nanoparticles. This work is presently in progress.

Third, as has been shown above, the inhomogeneous broadening due to the size distribution of nanocrystals does not exceed $200 \mathrm{meV}$. This value is a reasonable estimate for the peak width of the excitation spectrum for exciton luminescence. Consequently, it can be assumed that this peak is nothing but an envelope of the lowest $\mathrm{E}_{1 \mathrm{~S} 1 \mathrm{~s}}$-type transitions for different nanocrystals. The wider band in the excitation spectrum detected within the trap emission can then be associated with an envelope of transitions to higher lying energy levels. It is possible that carrier trapping is more effective from some excited electron or hole states with some special configurations of eigenfunctions. Inhibited relaxation between neighboring electron (hole) states due to acoustic phonon quantization ${ }^{40}$ promotes the capture of a nonequilibrium carrier. The smaller a nanoparticle is, the higher is the probability of this process. The observation can also be explained by exciton self-trapping in sufficiently small semiconductor nanoparticles; ${ }^{41}$ a critical factor is the possibility of exciton localization on a particular covalent bond which then leads to deformation of the capped nanocrystal. In this case, the difference in the excitation spectra, namely a shift by 50$100 \mathrm{meV}$, can result from an increase in the first excited energy level with a deformation of the nanoparticle. The exciton could possibly be localized on a bond between the crystallite core and a capping thiol group. ${ }^{42}$ This assumption is supported by the observation that degradation processes develop much faster when nanoparticles are in liquid solutions and when they are exposed to light.

B. Time-Resolved Luminescence. Luminescence decay kinetics provide additional important information on the recombination of photoinduced carriers in CdTe nanocrystals. We have found that luminescence decays of CdTe nanocrystals are wavelength-dependent, with the mean luminescence decay time 


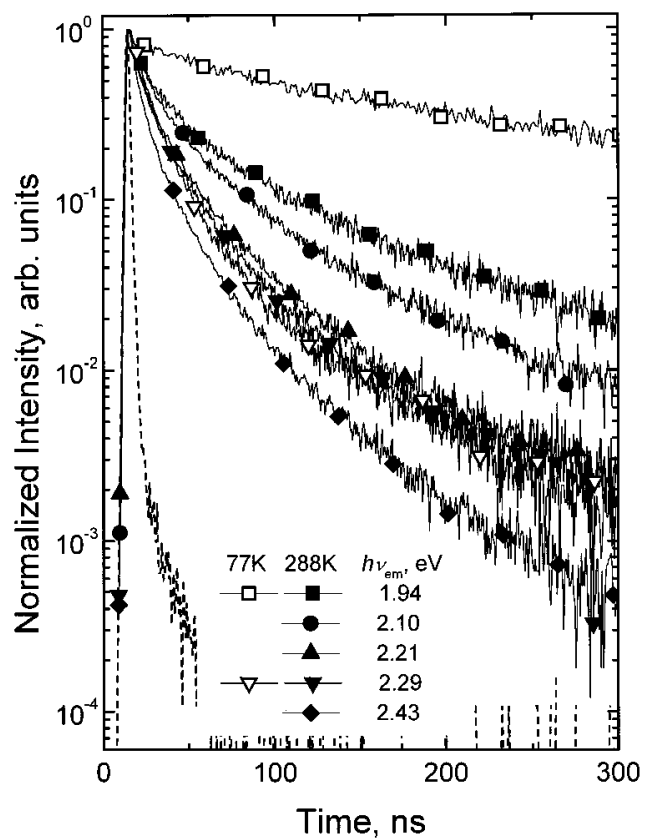

Figure 4. Room-temperature (solid symbols) and liquid-nitrogentemperature (open symbols) luminescence decay kinetics of CdTe nanocrystals detected at 1.94 (squares), 2.10 (circles), 2.21 (up triangles), 2.29 (down triangles), and $2.43 \mathrm{eV}$ (diamonds). The excitation pulse is shown for reference.

decreasing with increasing energy of the luminescence quantum (see inset in Figure 1 and Figure 4). Note the different temperature behavior of luminescence kinetics detected within the exciton and trap luminescence bands. All measured luminescence decays were found to be strongly nonexponential: both two- and three-exponential decay models failed to provide a satisfactory fit to the decay data yielding systematic plots of weighted residuals and $\chi^{2}$ values in the range of $1.25-1.61$.

Earlier, several luminescence models implying nonexponential decay kinetics were successfully used in an analysis of photoluminescence decay curves of semiconductor nanocrystals. The micellar kinetics ${ }^{43}$ originally developed to describe luminescence quenching in organic micelles implies a discrete number of traps per crystallite with a random distribution of traps over an ensemble of crystallites and is widely accepted as the relevant model for nonradiative dynamics in nanocrystals. ${ }^{44-46}$ Stretchedexponential decay laws imply migration of recombining components in a fractal space and can account for coexistence of local and migration lifetimes. These laws are inherent in a vast number of processes, ${ }^{47,48}$ including the photoluminescence of nanoparticles. ${ }^{49,50}$ However, our attempts to apply these models to the analysis of CdTe luminescence decays failed to provide both reasonably good fits to experimental decay kinetics and consistent spectral behavior of the parameters recovered.

Therefore, to provide quantitative information on the character of the photoluminescence decays of CdTe nanocrystals, we recovered decay time distributions from the luminescence decay curves. The decay time distribution analysis shows that CdTe luminescence decay kinetics involve processes with lifetimes ranging from a few hundred picoseconds to a few hundred nanoseconds (Figure 5). At present, we have no reasonable explanation for these extremely broad distributions of decay times describing photoluminescence decay kinetics of CdTe nanocrystals. This will be the subject of further investigations.

As has been pointed out above, the luminescence kinetics of the exciton and trap bands of CdTe nanocrystals show different temperature behaviors. Trap luminescence decay kinetics nor-

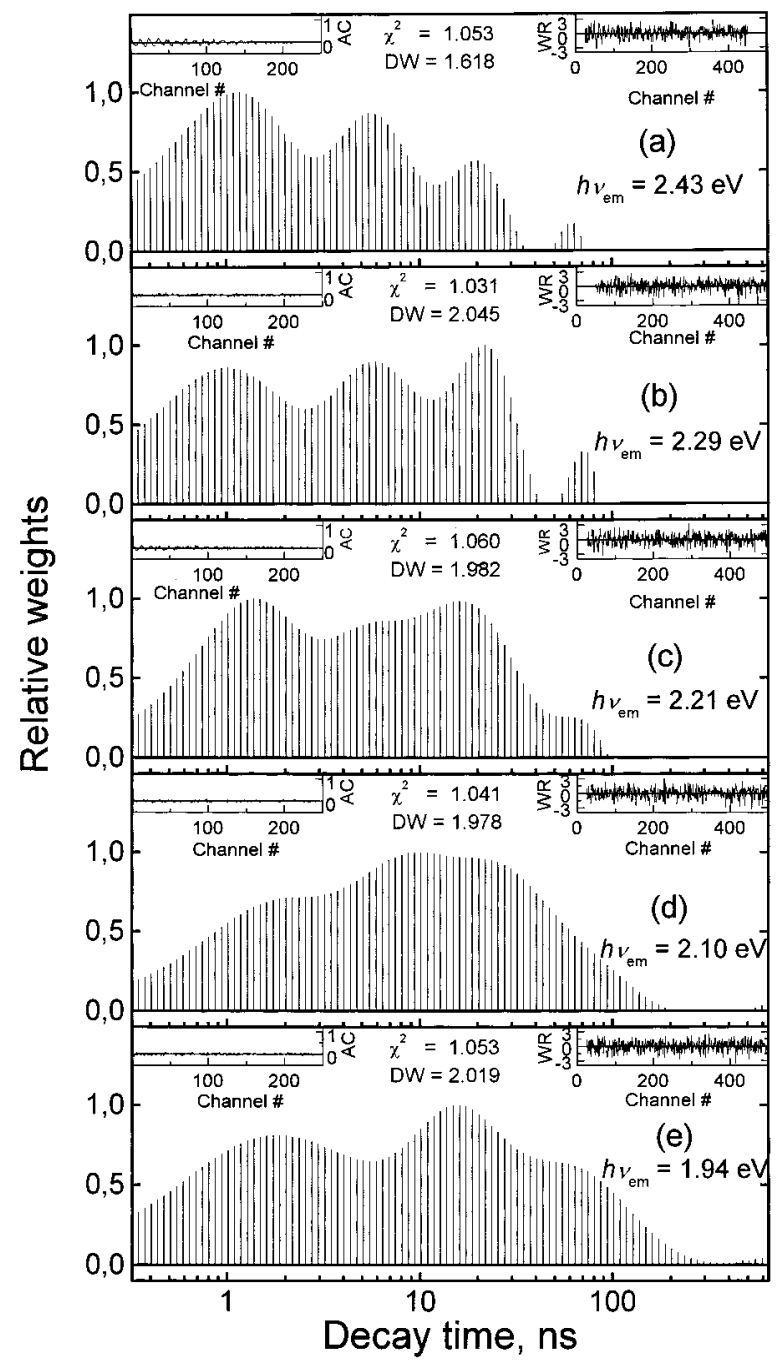

Figure 5. Decay time distributions recovered from the luminescence kinetics $(T=290 \mathrm{~K})$ of the exciton band detected at 2.43 (a), 2.29 (b), 2.21 (c), 2.10 (d), and $1.94 \mathrm{eV}$ (e). Insets show the corresponding plots of weighted residuals (WR) and their autocorrelation functions (AC), and values of the $\chi^{2}$ and DW parameters. 100 exponential terms with lifetimes uniformly distributed on the $\log \tau$ axis within the range of $0.32-640.0 \mathrm{~ns}$ were included in the decay analysis. 511 channels, 0.64 ns/channel.

mally determined by nonradiative deactivation of defect states show a strong temperature dependence becoming substantially faster at room temperature (see inset in Figure 1 and Figure 4). The thermal quenching of trap luminescence observed in the decay kinetics agrees with a decrease in the trap luminescence intensity with increasing temperature (Figure 1). The activation energy of the nonradiative recombination via trap states cannot exceed a few tens of millielectronvolts because this process completely dominates at $k_{B} T(T=290 \mathrm{~K}) \cong 25 \mathrm{meV}$ and starts to compete with the radiative processes only at $k_{B} T(T=77 \mathrm{~K})$ $\cong 6.6 \mathrm{meV}$. The recombination kinetics via the exciton channel is found to be identical at 77 and $290 \mathrm{~K}$. Whether this is indicative of the direct character of the recombination process or this finding is the result of competing processes involved in the deactivation scheme ${ }^{17}$ has still to be unraveled. A thorough temperature-dependent study of both the static and the timeresolved emission will help to answer this question. At least, we can state that the energy gap between the lowest allowed and forbidden excition states ${ }^{16,51}$ does not exceed the value of approximately $7 \mathrm{meV}$. 


\section{Conclusions}

In summary, steady-state and time-resolved luminescence studies have been reported for thiol-stabilized CdTe nanoparticles in aqueous solution. They possess the zinc blende crystal structure, with a mean size of the nanocrystals of $a \sim 2.4 \mathrm{~nm}$ which is substantially smaller than the exciton Bohr radius of $a_{\mathrm{B}}=7.3 \mathrm{~nm}$. The luminescence of the CdTe nanocrystals shows both a sharp exciton band and a wide band of emission via trap states. The trap luminescence band is substantially quenched when the temperature is increased from 77 to $290 \mathrm{~K}$, which manifests itself in both steady-state emission spectra and luminescence decay kinetics. Both the spectrum and luminescence decays of the exciton band are similar at 77 and $290 \mathrm{~K}$. Luminescence excitation spectra of CdTe nanocrystals reveal different maxima within the first absorption feature for detection within the exciton and trap emission bands. Higher energies of the exciting radiation quantum may promote a more efficient capture of the charge carriers by trap states. The luminescence decay kinetics of the CdTe nanocrystals depend on the emission wavelength, with the mean lifetime decreasing from $\sim 120 \mathrm{~ns}$ at $1.94 \mathrm{eV}$ to $\sim 20 \mathrm{~ns}$ at $2.43 \mathrm{eV}$. CdTe luminescence decays are strongly nonexponential and are described by extremely wide distributions of decay times spreading from a few hundred picoseconds to a few hundred nanoseconds.

Acknowledgment. This work was partially supported by the Volkswagen Foundation, Hannover. Thanks are due to Dr. M. Harrison for carefully reading the manuscript and for fruitful discussions.

\section{References and Notes}

(1) Brus, L. E. Appl. Phys. A 1991, 53, 465.

(2) Banyai, L.; Koch, S. W. Semiconductor Quantum Dots; World Scientific: Singapore, 1993.

(3) Spectroscopy of Isolated and Assembled Semiconductor Nanocrystals. Special Issue of J. Lumin. 1996, 70.

(4) Gaponenko, S. V. Optical Properties of Semiconductor Nanocrystals; Cambridge University Press: Cambridge, 1998.

(5) Woggon, U. Optical Properties of Semiconductor Quantum Dots; Springer-Verlag: Berlin, 1996.

(6) Klein, D. L.; Roth, R.; Lim, A. K. L.; Alivisatos, A. P.; McEuen, P. L. Nature 1997, 389, 699.

(7) Feldheim, D. L.; Keating, C. D. Chem. Soc. Rev. 1998, $28,1$.

(8) Colvin, V. L.; Schlamp, M. C.; Alivisatos, A. P. Nature 1994, 370, 354.

(9) Dabbousi, B. O.; Bawendi, M. G.; Onitsuka, O.; Rubner, M. F. Appl. Phys. Lett. 1995, 66, 1316.

(10) Gao, M.; Richter, B.; Kirstein, S.; Möhwald, H. J. Phys. Chem. B 1998, 102, 4096.

(11) Gaponenko, S. V.; Kapitonov, A. M.; Bogomolov, V. N.; Prokofiev, A. V.; Eychmüller, A.; Rogach, A. L. Pis'ma Zh. Eksp. Teor. Fiz. 1998, 68, 131 [JETP Lett. 1998, 68, 142].

(12) Henglein, A. Chem. Rev. 1989, 89, 1861.

(13) Weller, H. Adv. Mater. 1993, 5, 88.

(14) Kamat, P. V. Chem. Rev. 1993, 93, 267.

(15) Gao, M.; Kirstein, S.; Möhwald, H.; Rogach, A. L.; Kornowski, A.; Eychmüller, A.; Weller, H. J. Phys. Chem. B 1998, 102, 8360.

(16) Efros, Al. L.; Rosen, M.; Kuno, M.; Nirmal, M.; Norris, D. J.; Bawendi, M. Phys. Rev. B 1996, 54, 4843.

(17) Eychmüller, A.; Hässelbarth, A.; Katsikas, L.; Weller, H. Ber. Bunsen-Ges. Phys. Chem. 1991, 95, 79.

(18) Esch, V.; Flugel, B.; Khitrova, G.; Gibbs, H. M.; Jiajin, X.; Kang, K.; Koch, S. W.; Liu, L.-C.; Risbud, S. B.; Peyghambarian, N. Phys. Rev. B 1990, 42, 7450.
(19) Liu, L.-C.; Kim, M. J.; Risbud, S. H.; Carpenter, R. W. Philos. Mag. B 1991, 63, 769.

(20) Potter, B. G., Jr.; Simmons, J. H. J. Appl. Phys. 1990, 68, 1218. Ochoa, O. R.; Colajacomo, C.; Witkowski, E. J., III; Simmons, J. H.; Potter, B. G., Jr.; Solid State Commun. 1996, 98, 717.

(21) Neto, J. A. M.; Barbosa, L. C.; Cesar, C. L.; Alves, O. L.; Galembeck, F. Appl. Phys. Lett. 1991, 59, 2715. de Oliveira, R. C. M.; de Paula, A. M.; Plentz Fiho, F. O.; Neto, J. A. M.; Barbosa, L. C.; Alves, O. L.; Menezes, E. A.; Rios, J. M. M.; Fragnito, H. L.; Brito Cruz, C. H.; Cesar, C. L. Appl. Phys. Lett. 1995, 66, 439.

(22) Murray, C. B.; Norris, D. J.; Bawendi, M. G. J. Am. Chem. Soc. $1993,115,8706$.

(23) Bandaranayake, R. J.; Wen, G. W.; Lin, J. Y.; Jiang, H. X.; Sorensen, C. M. Appl. Phys. Lett. 1995, 67, 831.

(24) Rogach, A. L.; Katsikas, L.; Kornowski, A.; Su, D.; Eychmüller, A.; Weller, H. Ber. Bunsen-Ges. Phys. Chem. 1996, 100, 1772.

(25) Masumoto, Y.; Sonobe, K. Phys. Rev. B 1997, 56, 9734.

(26) Chemseddine, A.; Weller, H. Ber. Bunsen-Ges. Phys. Chem. 1993 97, 636.

(27) Evaluation of the "size" of the extremely small nanoparticles is not obvious: depending on the method used values of $1.9 \mathrm{~nm}$ (broadening of the diffraction peaks, Scherrer equation), $2.4 \mathrm{~nm}$ (position of the diffraction maximum in the small-angle region, Bragg equation), and 2.5 nm (HRTEM) were obtained. Generally, XRD underestimates and HRTEM overestimates the particle size. The nonspherical shape of CdTe nanoparticles also leads to difficulties in the size determination.

(28) O'Connor, D. V.; Phillips, D. Time-Correlated Single Photon Counting; Academic Press: London, 1984.

(29) Vecer, J.; Kowalczyk, A. A.; Dale, R. E. Rev. Sci. Instrum. 1993, 64, 3403.

(30) Siemiarczuk, A.; Wagner, B. D.; Ware, W. R. J. Phys. Chem. 1990 94, 1661. Gakamsky, D. M.; Goldin, A. A.; Petrov, E. P.; Rubinov, A. N. Biophys. Chem. 1992, 44, 47.

(31) Tikhonov, A. N.; Arsenin, V. Ya. Methods for Solving Ill-Posed Problems [in Russian], 3rd ed.; Nauka: Moscow, 1986.

(32) Petrov, E. P.; Kruchenok, J. V.; Rubinov, A. N. J. Fluoresc. 1999, $9,111$.

(33) Geladi, P.; Kowalski, B. R. Anal. Chim. Acta 1986, 185, 1.

(34) Golub, G. H.; Heath, M.; Wahba, G. Technometrics 1979, 21, 215 .

(35) Ostrowsky, N.; Sornette, D.; Parker, P.; Pike, E. R. Opt. Acta 1981, 28,1059 .

(36) Bawendi, M. G.; Wilson, W. L.; Rothberg, L.; Carroll, P. J.; Jedju, T. M.; Steigerwald, M. L.; Brus, L. E. Phys. Rev. Lett. 1990, 65, 1623.

(37) Woggon, U.; Gaponenko, S. V.; Langbein, W.; Klingshirn, C. Phys. Rev. B 1993, 47, 3684 .

(38) Kornowski, A.; Eichberger, R.; Giersig, M.; Weller, H.; Eychmüller, A. J. Phys. Chem. 1996, 100, 12467.

(39) Landolt-Börnstein, Numerical Data and Functional Relationships in Science and Technology; New Series, Group III, Vol. 22, Part a, Madelung, O., Ed.; Springer-Verlag: Berlin, 1987.

(40) Woggon, U.; Gaponenko, S. V.; Uhrig, A.; Langbein, W.; Klingshirn, C. Adv. Mater. Opt. Electron. 1994, 3, 141.

(41) Allan, G.; Delerue, C.; Lannoo, M. Phys. Rev. Lett. 1996, 76, 2961.

(42) Similar thiol-capped CdS nanoparticles were found to show certain exchange kinetics as revealed by NMR; i.e., thiol groups occasionally "leave" the semiconductor nanoparticles (unpublished).

(43) Gehlen, M. H.; De Schryver, F. C. Chem. Rev. 1993, 93, 199.

(44) Gaponenko, S. V.; Germanenko, I. N.; Petrov, E. P.; Stupak, A. P.; Bondarenko, V. P.; Dorofeev, A. M. Appl. Phys. Lett. 1994, 64, 85.

(45) Masumoto, Y.; Kawamura, T.; Ohzeki, T.; Urabe, S. Phys. Rev. B 1992, 46, 1827.

(46) Brus, L. E. J. Phys. Chem. 1994, 98, 3573.

(47) Kopelman, R. J. Stat. Phys. 1986, 42, 185; Science 1988, 241, 1620

(48) Lianos, P. J. Chem. Phys. 1988, 89, 5237.

(49) O’Neil, M.; Marohn, J.; McLendon, G. J. Phys. Chem. 1990, 94 4356.

(50) Gaponenko, S. V.; Petrov, E. P.; Woggon, U.; Wind, O.; Klingshirn, C.; Xie, Y. H.; Germanenko, I. N.; Stupak, A. P. J. Luminesc. 1996, 70, 364.

(51) Lefebvre, P.; Richard, T.; Mathieu, H.; Allégre, G. Solid State Commun. 1996, 98, 303. 